Communications in Physics, Vol.20, No. 4 (2010), pp. 295-300

\title{
ON THE SPIN LIQUID STATES OF THE FERMIONIZED HEISENBERG ANTIFERROMAGNETIC ON TRIANGULAR LATTICE
}

\author{
PHAM THI THANH NGA \\ Water Resources University \\ NGUYEN TOAN THANG \\ Institute of Physics, VAST
}

\begin{abstract}
We have analyzed the spin-1/2 isotropic Heisenberg antiferromagnetic system on triangular lattice within Popov-Fedotov functional intergral formalism when the spin operators are represented by the fermionic ones and the constraint of single occupancy conditions is taken into account by introducing an imaginary chemical potential. We study the effect of site occupation in quantum spin systems on the triangular lattice at finite temperature in a spin liquid states.
\end{abstract}

\section{INTRODUCTION}

The two dimensional spin-1/2 Heisenberg antiferromagnet (2DHAF) on a triangular lattice has generated much interrest in recent years [1]. The ideal nearest- neighbor Heisenberg antiferromagnet is given by the following Hamiltonian:

$$
H=\frac{1}{2} \sum_{i j} J_{i j} \vec{S}_{i} \vec{S}_{j},
$$

where the sum is over the nearest-neighbor pairs; $\vec{S}_{i}$ are spin- $1 / 2$ operators and $J_{i j}>0$.

Although the model describes experimental realizations only approximately, either due to anisotropies or because of additional interaction, the Hamiltonian (1) remains the principal starting point. One believes that in this model geometric frustration and low dimensionality would yield new physical phenomena. Early works on the triangular HAF $[2,3]$ suggested that the spin $-1 / 2$ ground state is a spin liquid, lacking long-range order (LRO). However, more recent studies have suggested that the $120^{\circ}$ magnetic structure remains stable even for $S=1 / 2[4,5]$. Yet other investigations support the original proposal of a disordered ground state at least at high energies [6]. Nevertheless, there is still a need to understand the conditions in which spin liquid phases exist and their phenomenological properties. From experimental point of view, recent studies on quantum magnets with a triangular lattice structure $\mathrm{Cs}_{2} \mathrm{CuCl}_{4}[7], \mathrm{K}-(\mathrm{ET})_{2} \mathrm{Cu}(\mathrm{CN})_{3}[8] \ldots$ show unusual properties that may be more or less understood in term of spin liquid states.

In describing spin liquid states of quantum magnets two different formalisms have been used. The first involved writing the spin as boson bilinear (Schwinger bosons) and using large- $\mathrm{N}$ approach $[9,10]$. The advantage of this approach is that it is able to access both magnetically ordered states arising from the condensation of the bosons as well as spin 
liquid states. A different way to describe spin liquid is to express the spin operators as a fermion bilinear $[11,12]$. Nevertheless, the representation of spin as a bilinear combination of Fermi or Bose operator enlarges the dimensionality of Hilbert space where these operator act. So the unphysical states should be excluded from the consideration resulting in a constraint requirement. Usually one replaces the local constraint on each point containing spins by so-called global constraint so that the restriction is fulfilled only in the average over all sites. Both fermionic and bosonic approaches are analytically pursued by writing down mean field ansatz where the constraint is imposed only on average to begin with. It is known that such a simplicity results in uncontrollable approximations for quantum spin, especially in low dimensions.

In new approachs for spin Hamiltonian free of the local constraint problem has been proposed by Popov and Fedotov [13]. Based on an exact representation of spin operators as fermion with imaginary chemical potential, the Popov-Fedotov representation resulted in the conventional Feynman temperature diagram technique, providing a rigorous treatment of the local constraint.

The goal of this report is to consider a spin liquid state for $2 \mathrm{D}$ spin $-1 / 2 \mathrm{HAF}$ on a triangular lattice, taking into account a strict site-occupation constraint.

\section{SPIN STATE MEAN FIELD EQUATIONS}

The Hamiltonian (1) can be projected onto Fock space by means of the transformation:

$$
\vec{S}_{i}=\frac{1}{2} a_{i \sigma}^{+} \vec{\sigma}_{\sigma \sigma^{\prime}} a_{i \sigma^{\prime}}
$$

where $\left\{a_{i \sigma}^{+}, a_{i \sigma}\right\}$ are anticommuting fermion operators with $\sigma= \pm 1 / 2, \vec{\sigma}$ are the Pauli matrices.

The main problem of expressing spin systems on Fock space is posed by the fact that Fock space always has a dimensionality higher than that for the spin space. Indeed, in

Fock space, each site can be occupied by 0,1 or 2 fermions corresponding to the states $|0,0\rangle,|1,0\rangle,|0,1\rangle,|1,1\rangle$, where $|0,0\rangle$ is the particle vacuum, $|1,0\rangle=|+1 / 2\rangle$; $|0,1\rangle=|-1 / 2\rangle$ and $|1,1\rangle=|+1 / 2,-1 / 2\rangle$ in terms of spin $1 / 2$ projections. In addition to physical states $|1,0\rangle$ and $|0,1\rangle$ two unphysical states $|1,1\rangle$ and $|0,0\rangle$ are introduced so one has to eliminate the contributions arising from empty sites and fully occupied sites. As it was first shown in [13], this could be achieved by introducing a single special valued imaginary chemical potential $\mu=-\mathrm{i} \pi / 2 \beta$ ( $\beta$ is inverse temperature). The partition function $\mathrm{Z}$ of the spin problem is given by:

$$
Z=(-i)^{N} \operatorname{Tr} \exp \left(-\beta\left(H_{F}-\mu \hat{N}_{F}\right),\right)
$$

where $\hat{H}_{F}$ is the operator obtained from (1) by the replacement (2) and:

$$
\hat{N}_{F}=\sum_{i=1}^{N}\left(a_{i \uparrow}^{+} a_{i \uparrow}+a_{i \downarrow}^{+} a_{i \downarrow}\right),
$$

where $N$ is the number of sites in the system. 
Nevertheless in the average over all states unphysical states cancel each other since $\operatorname{Tr}_{\text {unphys }}(\exp (\mathrm{i} \pi / 2))^{N_{F}}=(-i)^{o}+(-i)^{2}=0$. The representation (2) being of semionic origin results in the conventional Matsubara diagram technique with $\omega_{n}=\frac{2 \pi}{\beta}\left(n+\frac{1}{4}\right)$. We note that since auxiliary Fermi fields do not represent the true quasiparticle of the problem, helping only to treat properly the spin operators, the distribution function for these objects in general should not be a real function. $f(\varepsilon)=n(2 \varepsilon)+(i / 2) \operatorname{sech} \beta \varepsilon$, where $n(\varepsilon)$ is the standard Fermi distribution function. If observable quantities are calculated by first using operator identities valid in the constrained Hillbert space, some additional procedure is necessary to obtain the correct result [14].

In order to consider spin liquid phase we write the 2D spin - 1/2 HFM Hamiltonian given by (1) in terms of composite nonlocal operators $D_{i j}$ defined as $[9,15]$ :

$$
D_{i j}=\sum_{\sigma} a_{i \sigma}^{+} a_{j \sigma}
$$

With nearest neighbour interactions, the Hamiltonian takes the form:

$$
H_{F}=J \sum_{\langle i j\rangle}\left(\frac{1}{2} D_{i j}^{+} D_{i j}-\frac{n_{i}}{2}+\frac{n_{i} n_{j}}{4}\right),
$$

where the summation is taken on nearest neighbour pairs. As long as the exact site occupation constraint is satisfied the presence of the second and the third terms in (7) leads to a constraint quantity and hence are of no importance for the nature of the system. Thus we leave it out from the beginning. Following standard route and starting with the Hamiltonian:

$$
H_{F}=\frac{J}{2} \sum_{\langle i j\rangle} D_{i j}^{+} D_{i j}-\mu N_{F}
$$

We can express the partition function of the problem as a path integral over Grassmann variable $\left\{\eta_{i \sigma}^{*}, \eta_{i \sigma}\right\}$ corresponding to the operators $\left\{a_{i \sigma}^{+}, a_{i \sigma}\right\}$ defined above:

$$
Z=(-i)^{N} \int D \eta e^{\left.-\int_{0}^{\beta} d \tau\left\{\sum_{\alpha=(i \sigma)} \eta_{\alpha}^{*}(\tau) \partial_{\tau} \eta_{\alpha}^{(} \tau\right)+H_{F}\left(\eta_{\alpha}^{*}(\tau), \eta_{\alpha}(\tau)\right)\right\}} .
$$

The four-fermion interaction in (8) can be decomposed via a Hubbard-Stratonovich transformation by introducing the complex field $\chi_{i j}$ living on the links. The path integral (8) becomes then:

$$
Z=\frac{1}{i^{N} Z_{0}} \int D \chi D \eta e^{-S}
$$

where the action is:

$$
S=\int_{o}^{\beta} d \tau\left[\sum_{i \sigma} \eta_{i \sigma}^{*}\left(\partial_{\tau}-\mu\right) \eta_{i \sigma}+\frac{2}{J} \sum_{\langle i j\rangle} \chi_{i j}^{*} \chi_{i j}+\sum_{i j}\left(\chi_{i j}^{*} D_{i j}+D_{i j}^{*} \chi_{i j}\right)\right] .
$$

The fermion variables appear quadratically in $\mathrm{S}$ and are intergrated over freely. Thus we may intergrate out the Grassmann variables $\eta$ and obtain an effective action 
for $\chi_{i j}$. We decompose the fields $\chi_{i j}$ and their complex conjugates $\chi_{i j}^{*}$ into a mean field contribution and a fluctuation term:

$$
\chi_{i j}=\Delta_{i j}+\delta \chi_{i j}
$$

Since the mean field approximation describes the thermal equilibrium state we expect $\Delta_{i j}$ to be the time independent. As noted by Afflect and Marston [16], gauge invaviance of $\mathrm{S}$ implies invaviance under phase rotation of the $\eta_{i \sigma}$ with arbitrary space dependence $\eta_{i \sigma}(\tau)=\exp \left(i \theta_{i}\right) \eta_{i}(\tau)$, so the phase of $\Delta_{i j}$ transform as $\Delta_{i j} \rightarrow \Delta_{i j} \cdot e^{i\left(\theta_{i}-\theta_{j}\right)}$. The sum of the $\theta_{i j}=\theta_{i}-\theta_{j}$ around an elementary plaquette is gauge invariant so we may associate a "flux" to each plaquette according to

$$
\varphi \equiv \operatorname{Im} \ln \prod_{\langle i j\rangle \in \Delta} \Delta_{i j}
$$

where the product is over all links bordering the plaquette $\Delta$. In the triangular lattice each point has six nearest neighbours separated by the unit lattice vectors $\pm \vec{\delta}_{1} ; \quad \pm \vec{\delta}_{2} ; \pm \vec{\delta}_{3}$ :

$$
\vec{\delta}_{1}=(1,0) ; \vec{\delta}_{2}=\left(-\frac{1}{2}, \frac{\sqrt{3}}{2}\right) ; \vec{\delta}_{3}=\left(\frac{1}{2}, \frac{\sqrt{3}}{2}\right) \text {. }
$$

(the lattice constant is taken to be unity). The elementary plaquette is then a triangle defined as $\Delta \equiv\left(\vec{i}, \vec{i}+\vec{\delta}_{1}, \vec{i}+\vec{\delta}_{3}\right)$.

We anticipate that for each direction $\vec{\delta}_{\alpha}(\alpha=1,2,3)$ one has an directed bond

$$
\Delta_{i, i+\vec{\delta}_{\alpha}}=\Delta_{\alpha} e^{i \phi_{\alpha}}
$$

where $\Delta_{\alpha}$ are real. The configurations of the links $\Delta_{i j}$ are shown in Fig. 1.

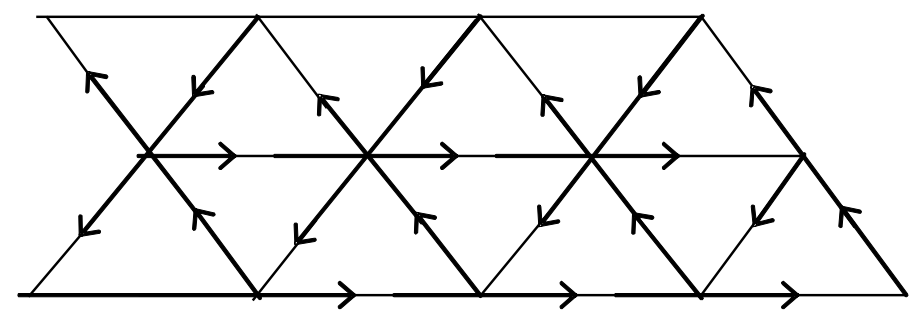

Fig. 1. Configurations of the links $\Delta_{i j}$ on the trianglar lattice

The mean-field free energy per site is found to be:

$$
F_{N F}=\frac{2}{J} \sum_{\alpha} \Delta_{\alpha}^{2}-\frac{1}{\beta N} \sum_{p} \ln 2 \operatorname{ch} \beta \varepsilon(p)+\frac{3}{4} J
$$

where

$$
\varepsilon(p)=2 \sum_{\alpha} \Delta_{\alpha} \cos \left(\phi_{\alpha}+\vec{\delta}_{\alpha} \vec{p}_{\alpha}\right)
$$


From (15) it is straightforward to obtain the mean field equations for $\Delta_{\alpha}$ by minimizing the free energy with respect to $\Delta_{\alpha}$

$$
\Delta_{\alpha}=\frac{J}{2 N} \sum_{p} \cos \left(\phi_{\alpha}+\vec{\delta}_{\alpha} \vec{p}\right) \tanh \beta \varepsilon(p)
$$

\section{PROPERTIES OF THE SELF - CONSISTENT SOLUTIONS}

Since one has three order parameters $\Delta_{\alpha}(\alpha=1,2,3)$ the various self-consistent solutions are possible.

First we consider solutions which have the rotational symmetry of the triangular lattice. In this case the following conditions for order parameters must be satisfied [17]

$$
\Delta_{\vec{\delta}_{1}}=e^{i \varphi} \Delta_{\vec{\delta}_{3}}=e^{2 i \varphi} \Delta_{\vec{\delta}_{2}}=e^{3 i \varphi} \Delta_{\vec{\delta}_{1}}
$$

Therefore $\phi=0, \frac{2 \pi}{3}$ and $\Delta_{1}=\Delta_{2}=\Delta_{3}$.

The phase with $\phi=0$ is an uniform zero flux state with $\varphi_{1}=\varphi_{2}=\varphi_{3}=0$.Numerical estimations of Eq. (15) and (17) give the ground state energy per site $E_{g r} \approx-0,25 \mathrm{~J}$ which is larger than the large-N calculation result for uniform zero - flux state $(-0,435 \mathrm{~J}$ [18]) and linear spin wave one for $\sqrt{3} \times \sqrt{3}$ Neel state $(-0,463 \mathrm{~J} \mathrm{[4]})$. The order parameter equation (18) gives the critical temperature $T_{c} \sim J / 2$. It is rather small in comparison with the Neel temperature estimated by linear spin wave theory [1]. Therefore our result do not support the uniform zero-flux state for isotropic HAF on the triangular lattice.

Next we consider self-consistent solutions which do not have the rotational symmetry of the triangular lattice. We have found that there are solutions where one or two of the order parameters vanishes. It is interesting to note that for boson mean field theory there is no solution when only one the order parameters is finite and the others are zero [17]. The solution with only one finite order parameter in our approach is quite acceptable having in mind a remarkable paper of Rochsar [19], who proved that the best saddle-point configuration on almost all lattices consists of a dimer covering of the lattice. That is $\Delta_{i j}$ is nonzero on one and only one of the links attached to each site. We do not analyze the other possible solutions because they have only academic interest. Neither we do make any comparison with experimental results because we are sure that no theory at mean - field level with the simplest isotropic HAF Hamiltonian can explain the rich physics behind the complex frustrated spin systems in real triangular lattice. At least we have to go beyond saddle point calculations to take into account fluctuations or to take additional interactions between spins expecting the theoretical results to fit experiments.

\section{CONCLUSIONS AND OUTLOOK}

In this report we have investigated the spin liquid state in the triangular lattice isotropic antiferromagnetic Heisenberg model by Popov-Fedotov semionic mean-field theory. We have got the self-consistent equations for free energy and order-parameters. We analyzed some particular solutions and found them rather resonable.

To our knowledge, it is the first attempt to study liquid state in triangular lattice by using semionic representation for spin operators where the local constraint is treated exactly. Although we have not got any new physics at mean - field level for a simplest 
HAF Hamiltonian, it seems straightforward to extend the formalism for more realistic Hamiltonian and to go beyond mean - field approximation to compare with experimental findings. Also it is intersting to see what the $\mathrm{SU}(2)$ and $Z_{2}$ lattice gauge theory for spin systems on triangular lattice looks like in the semionic approach.

\section{ACKNOWLEDGMENT}

This work is supported by NAFOSTED (No. 103.027809)

\section{REFERENCES}

[1] A. L. Chernyshev and M. E. Zhitomirsky, Phys. Rev. B79 (2009) 144416.

[2] P. W. Anderson, Mater. Res. Bull. 8 (1973) 153.

[3] V. Kalmeyer and R. B. Laughlin, Phys. Rev. Lett. 59 (1987) 2095.

[4] Th. Jolicocur and J. C. le Guillou, Phys. Rev. B40 (1989) 2227.

[5] A. V. Chubukov et al, J. Phys. Condens. Matter 6 (1994) 8891.

[6] W. Zheng et al., Phys. Rev. Lett. 96 (2006) 057201.

[7] R. Coldea et al., Phys. Rev. B68 (2003) 134424.

[8] Y Shimizu et al., Phys. Rev. B73 (2006) 140407.

[9] D. P. Arovas and A. Anerbach, Phys. Rev. B38 (1988) 316.

[10] Fa Wang and Ashvin Vishwanath, Phys. Rev. B74 (2006) 174423.

[11] A. A. Abrikosov, Physics 2 (1965) 5.

[12] X. G. Wen, Phys. Rev. B65 (2002) 1655113.

[13] V. N. Popov and S. A. Fedotov, Sov. Phys. JFTP 67 (1988) 535.

[14] J. Stein and R. Opperman, Z. Phys. B83 (1991) 333.

[15] R. Dillenschneider and J. Richert, Phys. Rev. B73 (2006) 24409.

[16] J. B. Marston and I. Afflect, Phys. Rev. B39 (1989) 11538.

[17] D. Yoshioka and J. Miyazaki, J. Phys. Soc. Jpn. 60 (1991) 614.

[18] S. Sachder, Phys. Rev. B45 (1992) 12377.

[19] D.S. Rochsar, Phys. Rev. B42 (1990) 2526. 